\title{
Urine AD7c-NTP Predicts Amyloid Deposition and Symptom of Agitation in Patients with Alzheimer's Disease and Mild Cognitive Impairment
}

\author{
Nan Zhanga,*, Liling Zhang ${ }^{\mathrm{a}}$, Yan $\mathrm{Li}^{\mathrm{a}}$, Marc L. Gordon ${ }^{\mathrm{b}}, \mathrm{Li} \mathrm{Cai}^{\mathrm{c}}$, Ying Wang ${ }^{\mathrm{c}}$, \\ Mengya Xing a and Yan Cheng ${ }^{\mathrm{a}}$ \\ ${ }^{a}$ Department of Neurology, Tianjin Medical University General Hospital, Tianjin Neurological Institute, \\ Tianjin, China \\ ${ }^{\mathrm{b}}$ The Litwin-Zucker Research Center, The Feinstein Institute for Medical Research, Manhasset, NY, USA \\ ${ }^{\mathrm{c}}$ PET/CT Center, Tianjin Medical University General Hospital, Tianjin, China
}

Handling Associate Editor: Jia Liu

Accepted 22 June 2017

\begin{abstract}
.
Background: Expression of neuronal thread protein (NTP), which is considered to be related to neuritic sprouting and neuronal death, may be elevated in brain tissue, cerebrospinal fluid, and even urine in patients with Alzheimer's disease (AD).

Objective: In this study, we analyzed the correlation between urine AD-associated NTP (AD7c-NTP) level, and amyloid- $\beta$ $(\mathrm{A} \beta)$ deposition, and clinical symptoms in $\mathrm{AD}$ and mild cognitive impairment (MCI).

Methods: Twenty-two patients with mild to moderate $\mathrm{AD}$ and 8 subjects with $\mathrm{MCI}$ were recruited. $\mathrm{A} \beta$ deposition was measured with $\left[{ }^{11} \mathrm{C}\right]$-labeled Pittsburgh compound B (PiB)-positron emission tomography (PET) in all participants. Urine AD7c-NTP levels were measured using enzyme-linked immunosorbent assay. Mini-Mental State Examination (MMSE) and Neuropsychiatric Inventory (NPI) were used to evaluate cognitive function and behavioral psychological symptoms, respectively.

Results: Fourteen (63.6\%) of AD patients and 2 (25.0\%) of MCI subjects were A $\beta$ positive on PiB-PET. There was a significant difference in urine AD7c-NTP level between $A \beta$ positive $(2.27 \pm 2.22 \mathrm{ng} / \mathrm{ml})$ and negative $(0.55 \pm 0.60 \mathrm{ng} / \mathrm{ml})$ subjects $(p=0.018)$. Using $1.46 \mathrm{ng} / \mathrm{ml}$ as a cut-off value, $68.8 \%$ of $\mathrm{A} \beta$ positive subjects showed elevated urine AD7c-NTP level, and 92.9\% of A $\beta$ negative subjects showed normal urine AD7c-NTP level. There were no relationships between urine AD7c-NTP level and MMSE and total NPI scores. However, AD7c-NTP level positively correlated with agitation score on NPI.

Conclusions: Urine AD7c-NTP had high specificity and moderate sensitivity in predicting A $\beta$ deposition among patients with cognitive impairment. Furthermore, urine AD7c-NTP level strongly correlated with the symptom of agitation.
\end{abstract}

Keywords: Agitation, Alzheimer's disease, amyloid- $\beta$, mild cognitive impairment, neuronal thread protein

\footnotetext{
${ }^{*}$ Correspondence to: Nan Zhang, MD, PhD, Department of Neurology, Tianjin Medical University General Hospital, 154, Anshan Road, Tianjin 300052, China. Tel.: +8622 60814504; Fax: +8622 60814592; E-mail: nkzhangnan@yeah.net.
}

\section{INTRODUCTION}

The neuropathological hallmarks of Alzheimer's disease $(\mathrm{AD})$ include extracellular amyloid plaques, intracellular neurofibrillary tangles principally 
comprised of hyperphosphorylated tau, and neuronal loss. Although amyloid- $\beta(\mathrm{A} \beta)$-positron emission tomography (PET) and measurement of $\mathrm{A} \beta_{42}$, total tau and phosphorylated tau concentration in cerebrospinal fluid (CSF) could increase accuracy in the diagnosis of $\mathrm{AD}$, the high cost and associated radiation exposure of PET, and the invasiveness of lumbar puncture, impede their clinical application. Therefore, an alternative biomarker for early diagnosis of $\mathrm{AD}$, which could be easily and safely obtained, would be desirable.

Neuronal thread protein (NTP), a family of membrane-spanning phosphoproteins, induces neuronal apoptosis and mitochondrial dysfunction, and is thought to be associated with neurodegenerationrelated neuritic sprouting and cell death [1-3]. AD-associated $41 \mathrm{kD} \mathrm{NTP} \mathrm{(AD7c-NTP)} \mathrm{was} \mathrm{isolated}$ by de la Monte and her colleagues from temporal lobe brain tissue of an $\mathrm{AD}$ patient [4], and was observed to be increased in brain tissue and CSF of patients with AD [4-6]. In addition, AD7c-NTP mRNA transcription was not detected in other organs or systems of human beings, such as lung, pancreas, liver, gastrointestinal tract, spleen, kidney, genital system, thyroid, or skeletal muscle. CSF levels of AD7c-NTP did not correlate with age, and were not obviously elevated in other central nervous system (CNS) diseases, such as Parkinson's disease (PD) or multiple sclerosis [4]. These results indicate that AD7c-NTP overexpression is specific to neuropathological processes in $\mathrm{AD}$ rather than nonspecific changes of aging or brain injury, and is rarely confounded by the status of other organs.

The size of AD7c-NTP protein is sufficiently small to be excreted into urine. Urine AD7c-NTP has been demonstrated to be elevated and to be stable over time in AD patients [7]. Furthermore, a meta-analysis of studies that compared urinary AD7c-NTP to standard clinical diagnosis found it to be a sensitive and specific test for the diagnosis of probable AD [8]. A previous urinary assay of AD7c-NTP (Alzheimer's disease reaction titer [ADRT]) was reported to have a sensitivity of $89-92.3 \%$ and specificity of $90-96.8 \%$ in detecting $\mathrm{AD}$ [9], although that assay failed to obtain approval by the FDA. Recently, a novel AD7cNTP enzyme-linked immunosorbent assay (ELISA) kit for urine testing has been developed in China. Compared with the old AD7c-NTP assay, which was developed by NYMOX using genetic engineering expression of whole-protein antibodies detection, the new kit detects specific antigenic determinants of two immunogenic polypeptide fragments of AD7cNTP. This new method improves the sensitivity in detecting AD7c-NTP by attenuating the impact of protein changes. The sensitivity and specificity of this testing kit has been reported to reach $89.3 \%$ and $84.7 \%$, respectively, in the diagnosis of $\mathrm{AD}$ (using National Institute of Neurological and Communicative Disorders and Stroke-Alzheimer's Disease and Related Disorders Association (NINCDS-ADRDA) criteria as the reference standard) [10]. It has been further demonstrated that urine AD7c-NTP level was increased even in mild cognitive impairment (MCI) individuals $[11,12]$. However, the correlation between urine AD7c-NTP and validated $\mathrm{AD}$ pathological biomarkers, such as $A \beta$, has not been investigated.

In this study, we evaluated urine AD7c-NTP level in the prediction of $A \beta$ deposition measured with Pittsburgh compound B (PiB)-PET, and further explored the relationship between AD7c-NTP level and severity of cognitive impairment and behavioral psychological symptoms in patients with probable or possible $\mathrm{AD}$ and MCI. In view of the close connection between tau pathology and both NTP overexpression and the symptom of agitation, we also analyzed the correlation between urine AD7c-NTP and agitation score on the Neuropsychiatric Inventory (NPI).

\section{MATERIALS AND METHODS}

\section{Participants}

This study was approved by the Ethics Committee of Tianjin Medical University General Hospital. Twenty-two mild to moderate AD patients and 8 MCI subjects were consecutively recruited from the memory clinic of Tianjin Medical University General Hospital. All participants underwent a comprehensive evaluation, including medical history, physical examination, clinical laboratory tests, brain magnetic resonance imaging (MRI), and neuropsychological assessment. AD patients met the diagnostic criteria of NINCDS-ADRDA for probable or possible $\mathrm{AD}$ [13]. MCI subjects met the recommendations of Petersen's MCI criteria [14], including cognitive decline with evidence from objective neuropsychological tasks (e.g., 1.5 or more standard deviations [SDs] below the norm), relatively preserved instrumental activities of daily living (ADL), as indicated 
by a modified 20-item ADL [15] score $\leq 22$, and not demented as indicated by a Clinical Dementia Rating (CDR) $[16] \leq 0.5$. Other inclusion criteria for $\mathrm{AD}$ and MCI included: (1) age range from 55 to 85; (2) AD patients with a Mini-Mental State Examination (MMSE) [17] score between 10 and 26, MCI individuals with a MMSE score $\geq 24$; (3) written informed consent was obtained. Exclusion criteria included: (1) a history or evidence from MRI of other CNS diseases, such as cerebrovascular disease, PD, multiple sclerosis, brain injury or brain tumor, etc.; (2) cognitive decline or dementia caused by any validated conditions other than $\mathrm{AD}$, such as, depression, abnormal thyroid function, drug or alcohol abuse, etc.; (3) urinary diseases or abnormal urinalysis $(\mathrm{pH}$, glucose, protein, cells or bacteria).

\section{Neuropsychological assessment}

All participants underwent neuropsychological assessment in the morning, administered by a trained investigator (L.Z.) who was blind to clinical conditions. The MMSE was used to assess cognitive function, with lower scores indicating poorer performance. The NPI [18] was used to evaluate behavioral and psychological symptoms, with higher scores indicating more severe and/or frequent symptoms. The total scores of MMSE and NPI and the item score of agitation on NPI were used for further analysis.

\section{PET imaging}

All participants underwent ${ }^{18} \mathrm{~F}$-labeled fluorodeoxyglucose (FDG)-PET and ${ }^{11} \mathrm{C}$-labeled PiBPET imaging, which were conducted at the PET/CT center of Tianjin Medical University General Hospital on a GE Discovery LS PET/CT scanner. PET images were acquired in 3D mode, yielding 35 slices with $4.25 \mathrm{~mm}$ thickness that covered the entire brain, and reconstructed to a $128 \times 128$ matrix $\left(2.5 \times 2.5 \mathrm{~mm}^{2}\right.$ pixel size $)$. Dynamic PiB-PET acquisitions that consisted of 34 frames $(4 \times 15 \mathrm{~s}$, $8 \times 30 \mathrm{~s}, 9 \times 60 \mathrm{~s}, 2 \times 180 \mathrm{~s}, 8 \times 300 \mathrm{~s}$, and $3 \times 600 \mathrm{~s})$ were acquired over $90 \mathrm{~min}$. The $60-90 \mathrm{~min} \mathrm{PiB}$ summation image was created and used for subsequent image analysis. ${ }^{11} \mathrm{C}-\mathrm{PiB}$ was administered as a bolus injection via antecubital vein, with a dose of 666-740 MBq. FDG-PET was conducted $1 \mathrm{~h}$ after PiB-PET scan. The subjects received an intravenous injection of 185-259 MBq FDG and remained in a darkened, quiet room. A 10-min static PET emission scan was performed 40 min after the FDG injection.

\section{PiB-PET data analysis}

The uptake of ${ }^{11} \mathrm{C}$-PiB was quantitated at the voxel level using the region-to-cerebellum ratio which is identical to the standardized uptake value ratio (SUVR). This simplified quantitation enables the utilization of a short 30-min image acquisition. Standardized region-of-interest (ROI) was defined on the MRI template image representing brain anatomy in accordance with the Montreal Neurological Institute (MNI) reference space. We merged and pooled subsets from the original Automated Anatomic Labeling (AAL) Atlas to form the following ROIs: middle frontal gyrus, medial prefrontal cortex, lateral temporal cortex, hippocampus and parahippocampus, inferior parietal lobe, posterior cingulate cortex and precuneus, striatum, thalamus, occipital lobe, superior temporal gyrus and supplementary motor area. Then, the preprocessing of $\mathrm{PiB}$ imaging data was performed using Statistical Parametric Mapping 8 (SPM8) on Matlab 2010b for Windows (Mathworks, Natick, MA). First, PiB integral images (data corrected for radioactive decay summed from $60 \mathrm{~min}$ to 90 min post injection) were created from the dynamic PET images (frames 32 to 34), and coregistered to the subject's MRI images. Second, MRI images were segmented into three classes (gray matter, white matter, and CSF) in SPM8 using 16 non-linear iterations and $7 \times 9 \times 7$ basis functions. Third, the PET images and the gray matter MRI images were normalized using a T1-weighted MRI template delivered with SPM to obtain normalization parameters. A gray matter probability map was created by applying a 0.5 threshold in MNI space, and coregistered to the AAL template. Then, PET counts were extracted from the gray matter probability map and the ROIs. Mean values for all regions were calculated from the integral PiB image. Target-to-cerebellum ratios were subsequently calculated for 11 bilateral regions. In this study, the ratio was considered as a dichotomous variable (cutoff value of 1.5 , determined through the upper $95 \%$ confidence interval (CI) from a cluster analysis of healthy individuals) to classify participants as belonging to the $\mathrm{PiB}$ positive or negative group. According to this analysis, 16 subjects (14 with $\mathrm{AD}$ and 2 with $\mathrm{MCI}$ ) were $\mathrm{PiB}$ positive, and the other 14 subjects ( 8 with $\mathrm{AD}$ and 6 with $\mathrm{MCI}$ ) were $\mathrm{PiB}$ negative. The PiB classification result was 
used as the gold standard for the receiver operating characteristic (ROC) analyses.

\section{Visual rating for FDG-PET images}

FDG-PET images were visually read by two experienced nuclear medicine physicians ( $\mathrm{Li} \mathrm{Cai}$ and Ying Wang) who were blind to the clinical data. The visual rating of FDG-PET followed descriptions by Rabinovici et al. [19]. Briefly, FDG frames for each subject were summed and normalized to mean activity in the pons, then were presented in the NIH color scale and could be windowed and viewed in 3 planes at the rater's discretion. FDGPET images were rated as "normal" if no obvious hypometabolism was observed, and as "frontotemporal dementia (FTD)" if hypometabolism was found mainly in frontal and/or anterior temporal cortex, and as "AD" if hypometabolism was mostly seen in temporoparietal cortex.

According to the FDG-PET readings, in the PiB negative group, $2 \mathrm{AD}$ patients and $3 \mathrm{MCI}$ subjects were rated as "normal", $3 \mathrm{AD}$ patients were rated as type "FTD", 1 AD patient and 1 MCI subject were rated as "AD", and the other 4 subjects showed nonspecific hypometabolism. All PiB positive subjects were rated as "AD" on FDG-PET readings. The sensitivity, specificity, positive predictive value (PPV) and negative predictive value (NPV) of FDG-PET for predicting $\mathrm{PiB}$ positivity were $100 \%$ (95\% CI 75.9-100), 85.7\% (95\% CI 56.2-97.5), 88.9\% (95\% CI 63.9-98.1), and 100\% (95\% CI 69.9-100), respectively, in this study. Figure 1 shows representative PiB-PET, FDG-PET, and T1-weighted MRI images of four participants from this study.

Urinary AD7c-NTP assay

Ten $\mathrm{ml}$ samples of urine (8 samples were random urine, the others were first morning urine) were collected from each participant, and then were immediately processed or refrigerated at $4{ }^{\circ} \mathrm{C}$ for up to 7 days. AD7c-NTP concentration was assayed using an ELISA kit (Anqun Biological Technology Co. Ltd., Shenzhen, China). According to the instructions for the kit, the urine sample was placed at room temperature for $20 \mathrm{~min}$ before performing the assay. The procedure is as follows: (1) $100 \mu \mathrm{l}$ of the urine specimen was put into the precoated plate and incubated at $37^{\circ} \mathrm{C}$ for $1 \mathrm{~h}$; (2) then the plate was loaded with $100 \mu \mathrm{l}$ biotinylated rabbit anti-AD7c-NTP antibody and incubated at $37^{\circ} \mathrm{C}$ for $1 \mathrm{~h}$; (3) the plate was loaded

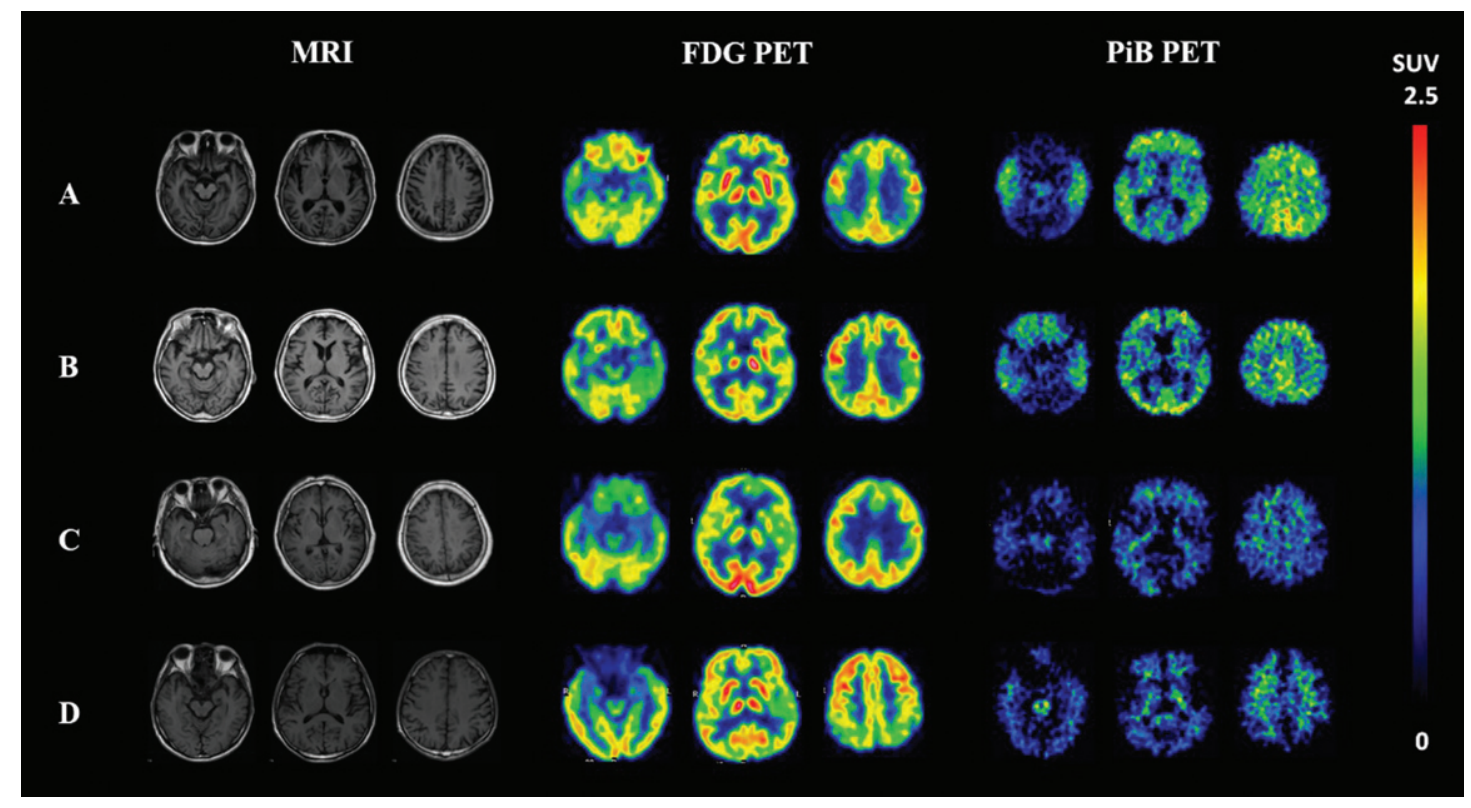

Fig. 1. T1-weighted MRI, FDG-PET and PiB-PET images of four representative participants. A 64-year-old female AD patient with positive $\mathrm{A} \beta$ deposition on PiB-PET and "AD" type of hypometabolism on FDG-PET (A); a 76-year-old female MCI subject with positive A $\beta$ deposition on PiB-PET and "AD" type of hypometabolism on FDG-PET (B); a 78-year-old female AD patient with negative PiB and "FTD" type of hypometabolism (C); and a 71-year-old male AD patient with negative PiB and non-specific hypometabolism on FDG-PET (D). FDG, fluorodeoxyglucose; PiB, Pittsburgh compound B; PET, positron emission tomography; AD, Alzheimer's disease; MCI, mild cognitive impairment; FTD, frontotemporal dementia. 
with $100 \mu \mathrm{l}$ of horseradish peroxidase labeled avidin and incubated at $37^{\circ} \mathrm{C}$ for $30 \mathrm{~min}$. Each of the above steps was followed by washing with PBS 5 times. (4) $50 \mu \mathrm{l}$ of chromogenic reagent $\mathrm{A}$ and $\mathrm{B}$ was added in turn and incubated at $37^{\circ} \mathrm{C}$ for $15 \mathrm{~min}$; (5) the reaction was stopped by adding $50 \mu \mathrm{l}$ of sulfuric acid as stop buffer. The optical density (OD) value of the reaction product was measured at $450 \mathrm{~nm}(630 \mathrm{~nm}$ as a reference) with Varioskan ${ }^{\circledR}$ Flash Spectral Scanning Multimode Readers (Thermo Fisher Scientific, Waltham, MA, USA) within 15 min after stopping the reaction. The AD7c-NTP concentration in a urine specimen is positively correlated with the OD value and was calculated according to the standard curve of recombinant human AD7c-NTP peptides measured at the same time.

\section{Statistical analysis}

Statistical analyses were carried out with SPSS 13.0 (SPSS Inc., USA). The comparison of demographic and clinical variables between the PiB positive group and the $\mathrm{PiB}$ negative group was performed using independent-sample $t$ test and Pearson chi-square tests where appropriate. Taking into account the age and sex effects that have been observed in a previous study using the same test kit [20], the difference in urine AD7c-NTP level between the two groups was conducted in a general linear model using age and sex as covariates. Sensitivity and specificity of urine AD7c-NTP were calculated using ROC curve with binary assessment of PiBPET (positive or negative) as the reference standard. Partial correlation analysis was performed to assess the relationship between urine AD7c-NTP concentration and MMSE and NPI total scores, and subscales of the NPI, using age, sex, and disease duration as covariates. All the tests were two-tailed, and values of $p<0.05$ were regarded as statistically significant.

\section{RESULTS}

\section{Demographic and clinical characteristics}

Table 1 describes the demographic and clinical characteristics of all participants. There were no significant differences in age, sex, years of education, disease duration, and NPI score between the PiB positive and negative subjects. MMSE score in the PiB negative group was higher than in the $\mathrm{PiB}$ positive group $(p<0.05)$. This is attributable to the different ratio of $\mathrm{AD}$ and $\mathrm{MCI}$ between the $\mathrm{PiB}$ positive
Table 1

Demographic and clinical characteristics of all participants

\begin{tabular}{lccc}
\hline & $\begin{array}{c}\text { PiB positive } \\
(n=16)\end{array}$ & $\begin{array}{c}\text { PiB negative } \\
(n=14)\end{array}$ & $p$ value \\
\hline Diagnosis (MCI/AD) & $2 / 14$ & $6 / 8$ & 0.061 \\
Age (y) & $66.75 \pm 9.33$ & $65.86 \pm 7.61$ & 0.778 \\
Sex (Female/Male) & $13 / 3$ & $8 / 6$ & 0.151 \\
Years of education (y) & $9.88 \pm 3.91$ & $11.50 \pm 4.11$ & 0.277 \\
Disease duration (y) & $2.38 \pm 1.03$ & $1.93 \pm 0.73$ & 0.186 \\
MMSE & $17.63 \pm 5.39$ & $22.11 \pm 6.04$ & 0.036 \\
NPI & $13.44 \pm 7.62$ & $10.93 \pm 5.46$ & 0.315 \\
\hline
\end{tabular}

Values are mean $\pm \mathrm{SD}$ unless otherwise indicated. $\mathrm{AD}$, Alzheimer's disease; MCI, mild cognitive impairment; MMSE, Mini-Mental State Examination; NPI, Neuropsychiatric Inventory; PiB, Pittsburgh compound B.

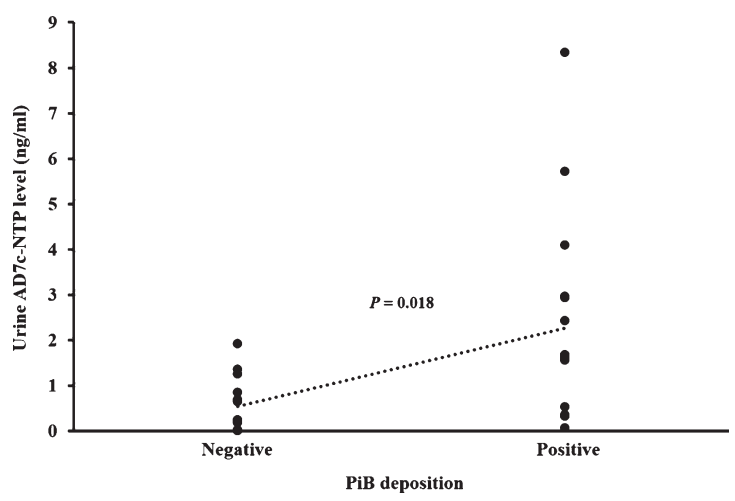

Fig. 2. Difference in urine AD7c-NTP level between PiB positive subjects and $\mathrm{PiB}$ negative subjects. The mean values of urine AD7c-NTP are $2.27 \pm 2.22 \mathrm{ng} / \mathrm{ml}$ in PiB positive group $(n=16)$ and $0.55 \pm 0.60 \mathrm{ng} / \mathrm{ml}$ in PiB negative group $(n=14)$.

group (AD/MCI: 14/2) and the $\mathrm{PiB}$ negative group (AD/MCI: 8/6).

\section{Difference in urine AD7c-NTP level between PiB positive and negative subjects}

The urine AD7c-NTP concentrations were $1.78 \pm 2.06 \mathrm{ng} / \mathrm{ml}$ and $0.62 \pm 0.73 \mathrm{ng} / \mathrm{ml}$ in $\mathrm{AD}$ patients and MCI subjects, respectively. Urine AD7c-NTP level was prominently higher in the PiB positive group $(2.27 \pm 2.22 \mathrm{ng} / \mathrm{ml})$ than in the $\mathrm{PiB}$ negative group $(0.55 \pm 0.60 \mathrm{ng} / \mathrm{ml})$ (Fig. 2). This difference was still present after adjusting for age and sex $(p=0.018)$. The optimal cutoff value was calculated based on the maximum sum of sensitivity and specificity using ROC curve (Fig. 3). At a cutoff of $1.46 \mathrm{ng} / \mathrm{ml}$, there were 11 patients $(68.8 \%)$ with elevated AD7c-NTP in the PiB positive group, and 13 patients (92.9\%) with normal AD7c-NTP in the $\mathrm{PiB}$ negative group. The PPV and NPV of urine 


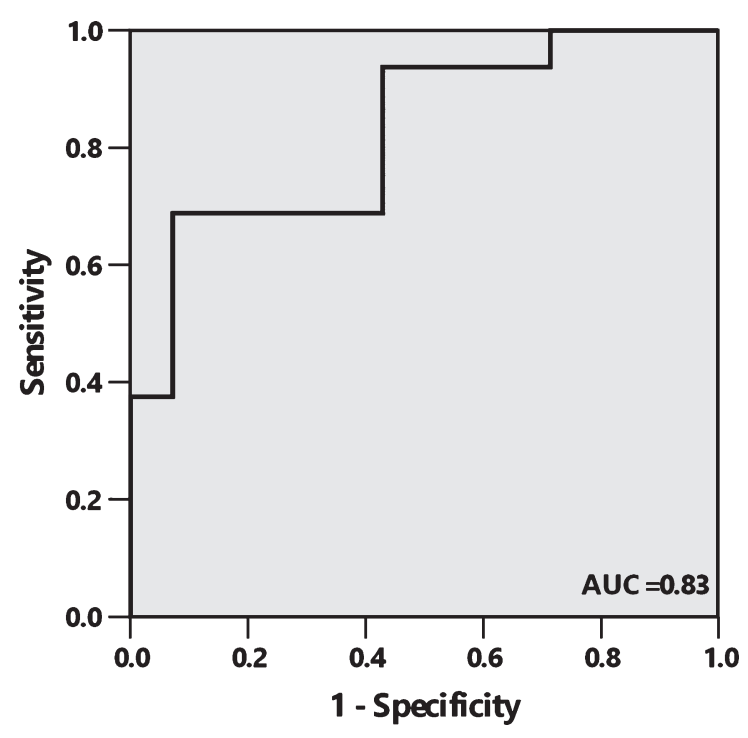

Fig. 3. Receiver operating characteristic (ROC) curve depicts sensitivity and 1 minus specificity level of urine AD7c-NTP for discrimination between $\mathrm{PiB}$ positive and $\mathrm{PiB}$ negative patients with $\mathrm{AD}$ or MCI. The area under the curve (AUC) is $0.83(95 \%$ CI 0.68 to 0.97 ), with sensitivity, specificity, PPV, and NPV of $68.8 \%$ (95\% CI 41.5-87.9), 92.9\% (95\% CI 64.2-99.6), 91.7\% (95\% CI 59.8-99.6), and 72.2\% (95\% CI 46.4-89.3), respectively, at a cutoff of $1.46 \mathrm{ng} / \mathrm{ml}$.

AD7c-NTP in predicting PiB deposition were 91.7\% (95\% CI 59.8-99.6) and 72.2\% (95\% CI 46.4-89.3), respectively.

\section{The relationship between urine $A D 7 c-N T P$}

and cognitive decline and behavioral

and psychological symptoms

In all participants, MMSE and NPI total scores did not correlate with urine AD7c-NTP level after controlling for age, sex, and disease duration. The agitation subscale score on NPI positively correlated with AD7c-NTP level $(p<0.001)$. This relationship was confirmed by comparison of AD7c-NTP level between subjects with agitation (score $>1$ ) and subjects without agitation (score of 0 or 1 ), using disease duration as a covariate. In this comparison, subjects with agitation showed higher NTP levels than subjects without agitation did $(p=0.004)$ (Fig. 4). Because only mild to moderate $\mathrm{AD}$ patients and MCI subjects were recruited in this study, several items on the NPI were absent (score $<2$ ) in most participants (more than 80\%), such as hallucinations, delusions, euphoria, disinhibition, aberrant motor behavior, and appetite and eating abnormalities. For other subscales of the NPI, including depression,

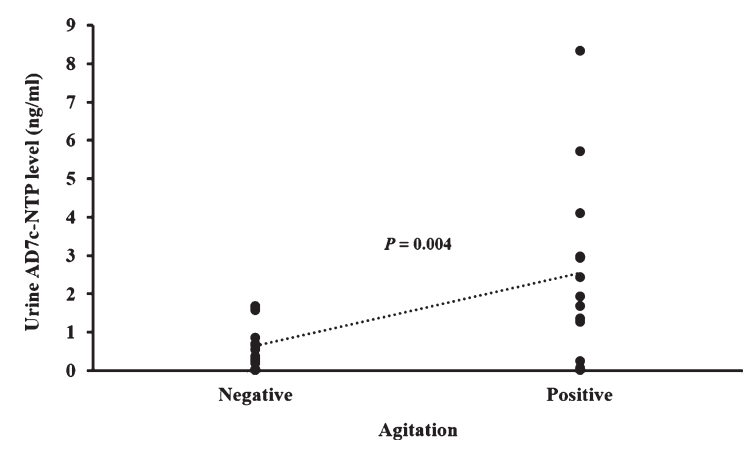

Fig. 4. Difference of AD7c-NTP level between subjects with agitation and subjects without agitation. Agitation with score of 2 or more in NPI was considered as a reliable symptom. Using this cutoff value, participants were divided into agitation positive group and agitation negative group. The urine AD7c-NTP levels are $2.54 \pm 2.39 \mathrm{ng} / \mathrm{ml}$ in agitation positive subjects $(n=13)$ and $0.65 \pm 0.61 \mathrm{ng} / \mathrm{ml}$ in agitation negative subjects $(n=17)$.

anxiety, apathy, irritability, and nighttime behavior disturbances, we did not find any correlation with AD7c-NTP level.

\section{DISCUSSION}

According to our knowledge, this is the first study designed to verify the concordance between urine AD7c-NTP and PiB-PET. In this study, we demonstrated that urine AD7c-NTP was elevated in AD and MCI patients with brain $A \beta$ deposition compared with those without $\mathrm{A} \beta$ deposition. Using a cutoff of $1.46 \mathrm{ng} / \mathrm{ml}$, the sensitivity, specificity, PPV, and NPV of urine AD7c-NTP in predicting A $\beta$ deposition were $68.8 \%$ (95\% CI 41.5-87.9), 92.9\% (95\% CI 64.2-99.6), 91.7\% (95\% CI 59.8-99.6), and $72.2 \%$ (95\% CI 46.4-89.3), respectively. We further observed a correlation between urine AD7c-NTP and agitation, but not cognitive function in patients with $\mathrm{AD}$ and MCI.

It has been reported that the sensitivity and specificity of the urine AD7c-NTP test reached approximately $80-89 \%$ and $90-91 \%$, respectively, for differentiating $\mathrm{AD}$ patients from non-AD dementia and non-dementia controls $[21,22]$. The high sensitivity and specificity of urine AD7c-NTP in AD diagnosis have also been demonstrated in multicenter studies [23, 24], although there were $30 \%$ of PD patients with high AD7c-NTP level [24]. Moreover, the urine testing kit used in this study has been validated in patients with $\mathrm{AD}$ and $\mathrm{MCI}$ within the Chinese population [10-12]. However, as no reliable biomarkers were available, there could 
have been considerable heterogeneity in etiology and neuropathology of clinically diagnosed AD and MCI patients in previous studies. According to the National Institute on Aging and the Alzheimer's Association (NIA-AA) recommendations, incorporating biomarkers, such as $A \beta$ PET, indicates a high likelihood of the diagnosis in $\mathrm{AD}$ or MCI due to $\mathrm{AD}$ $[25,26]$. Thus, it is necessary to evaluate the accuracy of AD7c-NTP in diagnosing AD and MCI based on AD pathological biomarkers.

${ }^{11} \mathrm{C}$-PiB tracer binds with high affinity to $\mathrm{A} \beta$, which is one of the main hallmarks of $\mathrm{AD}$ neuropathology. It was estimated that $A \beta$ deposition measured with PiB-PET precedes hippocampal atrophy and memory impairment in a prospective study [27]. Neuropathological validation studies of PiB-PET demonstrated that both qualitative (with a pooled sensitivity of $73 \%$ and pooled specificity of $100 \%$ ) and quantitative (with a pooled correlation coefficient of $r=0.88$ ) approaches have good concordance with histopathological results of amyloid deposition [28]. When using PiB-PET as a gold standard, we found the sensitivity of urine AD7c-NTP to be slightly lower than in previous studies, although the specificity remained high in this study. This result indicates that although AD7c-NTP expression was found to be independent of $A \beta$ accumulation in vitro [1], urine AD7c-NTP level could predict A $\beta$ deposition in AD and MCI patients with a PPV of $91.7 \%$ (95\% CI 59.8-99.6) and a NPV of $72.2 \%$ (95\% CI 46.4-89.3).

Although negative correlations between CSF AD7c-NTP concentration and MMSE or Blessed dementia scale scores have been reported [4, 6], whether urine AD7c-NTP could reflect disease severity is still controversial. One study reported higher urine AD7c-NTP levels in advanced AD patients, compared to patients at early stage [22]. Conversely, in another study, moderate AD patients with CDR score of 2 had a lower urine AD7c-NTP level than mild AD patients with CDR score of 1 [24]. In our present study, we did not find a specific correlation between urine AD7c-NTP level and MMSE score. First, not all our participants suffered from $\mathrm{AD}$ or $\mathrm{MCI}$ due to $\mathrm{AD}$, especially in $\mathrm{A} \beta$ negative subjects. There is no evidence of correlation between AD7c-NTP and cognition or behavioral psychological symptoms in healthy subjects or other types of dementia. Furthermore, neurodegenerationrelated neuritic sprouting reflected by AD7c-NTP may decompensate in the progression of AD. Therefore, the dynamic changes in AD7c-NTP level as AD neuropathology evolves over the course of the disease needs further investigation.

Interestingly, although there was no relationship between urine AD7c-NTP level and NPI total score, we found that urine AD7c-NTP level positively correlated with the symptom of agitation. Tau pathology is considered to be closely related to clinical symptoms in $\mathrm{AD}$, especially agitation, which has been found to be associated with the presence of neurofibrillary tangles in frontal cortex [29]. Furthermore, AD7c-NTP was observed to positively correlate with phosphorylated tau accumulation in neuronal cells $[2,30]$ and CSF concentration of tau in AD patients [6]. Taken together, our results indirectly support a relationship between AD7c-NTP and tau pathology in AD.

\section{Limitations}

First, reliable detection and quantification of urine AD7c-NTP requires that the urine should be sterile and free of particulate debris. In this study, 8 samples were collected as random urine instead of first morning urine, because most of our participants were not inpatient. It is possible that non-first morning urine samples may cause false negatives, because of nonspecific analyte dilution from urinary dilution. This could be a reason for lower sensitivity in our study compared to previous studies. Second, this is a preliminary study using a validated biomarker to evaluate the potential utility of the urine AD7c-NTP assay. Any findings from this study may be limited by the small sample size. Third, we only included clinically diagnosed AD and MCI participants in this study. Although the specificity and PPV are high in distinguishing $\mathrm{AD}$ and $\mathrm{MCI}$ patients with $\mathrm{A} \beta$ deposition from those without $A \beta$ deposition, the ability of urine AD7c-NTP to discriminate between AD and other types of dementia or neurodegenerative diseases, such as vascular dementia, FTD and PD, is still under investigation. Finally, although all patients with positive PiB-PET scans were also confirmed by FDG-PET readings, there were still $2 \mathrm{PiB}$ negative subjects who were rated as AD on FDG-PET readings. Therefore, the clinical utility of urine AD7cNTP needs further validation using histopathology as a gold standard.

\section{Conclusions}

Urine AD7c-NTP had a moderate sensitivity and high specificity in predicting $A \beta$ deposition in clinically diagnosed $\mathrm{AD}$ and MCI patients. Furthermore, 
urine AD7c-NTP level strongly correlated with the symptom of agitation.

\section{ACKNOWLEDGMENTS}

This work was supported in part by the National Natural Science Foundation of China (grant number 81500926) and the National Key Clinical Specialty Construction Project of China.

Authors' disclosures available online (http://j-alz. com/manuscript-disclosures/17-0383r1).

\section{REFERENCES}

[1] De La Monte SM, Wands JR (2001) The AD7c-NTP neuronal thread protein biomarker for detecting Alzheimer's disease. J Alzheimers Dis 3, 345-353.

[2] de la Monte SM, Wands JR (2001) Alzheimer-associated neuronal thread protein-induced apoptosis and impaired mitochondrial function in human central nervous systemderived neuronal cells. J Neuropathol Exp Neurol 60, 195-207.

[3] de la Monte SM, Wands JR (2001) Neurodegeneration changes in primary central nervous system neurons transfected with the Alzheimer-associated neuronal thread protein gene. Cell Mol Life Sci 58, 844-849.

[4] Monte SM, Ghanbari K, Frey WH, Beheshti I, Averback P, Hauser SL, Ghanbari HA, Wands JR (1997) Characterization of the AD7C-NTP cDNA expression in Alzheimer's disease and measurement of a $41-\mathrm{kD}$ protein in cerebrospinal fluid. J Clin Invest 100, 3093-3104.

[5] De La Monte SM, Carlson RI, Brown NV, Wands JR (1996) Profiles of neuronal thread protein expression in Alzheimer's disease. J Neuropathol Exp Neurol 55, 10381050.

[6] Kahle PJ, Jakowec M, Teipel SJ, Hampel H, Petzinger GM, Di Monte DA, Silverberg GD, Moller HJ, Yesavage JA, Tinklenberg JR, Shooter EM, Murphy GM Jr (2000) Combined assessment of tau and neuronal thread protein in Alzheimer's disease CSF. Neurology 54, 1498-1504.

[7] Levy S, McConville M, Lazaro GA, Averback P (2007) Competitive ELISA studies of neural thread protein in urine in Alzheimer's disease. J Clin Lab Anal 21, 24-33.

[8] Zhang J, Zhang CH, Li RJ, Lin XL, Chen YD, Gao HQ, Shi SL (2014) Accuracy of urinary AD7c-NTP for diagnosing Alzheimer's disease: A systematic review and meta-analysis. J Alzheimers Dis 40, 153-159.

[9] Goodman IJ (2008) Practical utility of urinary assay in the diagnosis of Alzheimer's disease: AlzheimAlert. Expert Rev Mol Diagn 8, 21-28.

[10] Ma L, Wang R, Han Y, Sheng S, Zhu J, Ji Z, Zhao Z, Cao Z, Wang P (2015) Development of a novel urine Alzheimer-associated neuronal thread protein ELISA kit and its potential use in the diagnosis of Alzheimer's disease. J Clin Lab Anal 30, 308-314.

[11] Ma L, Chen J, Wang R, Han Y, Zhang J, Dong W, Zhang X, Wu Y, Zhao Z (2015) The level of Alzheimer-associated neuronal thread protein in urine may be an important biomarker of mild cognitive impairment. J Clin Neurosci 22, 649-652.
[12] Wang C, Cui Y, Yang J, Zhang J, Yuan D, Wei Y, Li Y, Duo Y, Li S, Zhu W, Zheng L (2015) Combining serum and urine biomarkers in the early diagnosis of mild cognitive impairment that evolves into Alzheimer's disease in patients with the apolipoprotein E 4 genotype. Biomarkers 20, 84-88.

[13] McKhann G, Drachman D, Folstein M, Katzman R, Price D, Stadlan EM (1984) Clinical diagnosis of Alzheimer's disease: Report of the NINCDS-ADRDA Work Group under the auspices of Department of Health and Human Services Task Force on Alzheimer's Disease. Neurology 34, 939-944.

[14] Winblad B, Palmer K, Kivipelto M, Jelic V, Fratiglioni L, Wahlund LO, Nordberg A, Backman L, Albert M, Almkvist O, Arai H, Basun H, Blennow K, de Leon M, DeCarli C, Erkinjuntti T, Giacobini E, Graff C, Hardy J, Jack C, Jorm A, Ritchie K, van Duijn C, Visser P, Petersen RC (2004) Mild cognitive impairment-beyond controversies, towards a consensus: Report of the International Working Group on Mild Cognitive Impairment. J Intern Med 256, 240-246.

[15] Lawton MP, Brody EM (1969) Assessment of older people: Self-maintaining and instrumental activities of daily living. Gerontologist 9, 179-186.

[16] Berg L (1988) Clinical Dementia Rating (CDR). Psychopharmacol Bull 24, 637-639.

[17] Folstein MF, Folstein SE, McHugh PR (1975) “Mini-mental state". A practical method for grading the cognitive state of patients for the clinician. J Psychiatr Res 12, 189-198.

[18] Cummings JL, Mega M, Gray K, Rosenberg-Thompson S, Carusi DA, Gornbein J (1994) The Neuropsychiatric Inventory: Comprehensive assessment of psychopathology in dementia. Neurology 44, 2308-2314.

[19] Rabinovici GD, Rosen HJ, Alkalay A, Kornak J, Furst AJ, Agarwal N, Mormino EC, O'Neil JP, Janabi M, Karydas A, Growdon ME, Jang JY, Huang EJ, Dearmond SJ, Trojanowski JQ, Grinberg LT, Gorno-Tempini ML, Seeley WW, Miller BL, Jagust WJ (2011) Amyloid vs FDG-PET in the differential diagnosis of AD and FTLD. Neurology 77, 2034-2042.

[20] Ma L, Chen J, Wang R, Han Y, Zhang J, Dong W, Zhao Z, Liu Y, Chu X (2014) Alzheimer-associated urine neuronal thread protein level increases with age in a healthy Chinese population. J Clin Neurosci 21, 2118-2121.

[21] Ghanbari H, Ghanbari K, Beheshti I, Munzar M, Vasauskas A, Averback P (1998) Biochemical assay for AD7C-NTP in urine as an Alzheimer's disease marker. J Clin Lab Anal 12, 285-288.

[22] Munzar M, Levy S, Rush R, Averback P (2002) Clinical study of a urinary competitve ELISA for neural thread protein in Alzheimer disease. Neurol Clin Neurophysiol 2002, $2-8$.

[23] Goodman I, Golden G, Flitman S, Xie K, McConville M, Levy S, Zimmerman E, Lebedeva Z, Richter R, Minagar A, Averback P (2007) A multi-center blinded prospective study of urine neural thread protein measurements in patients with suspected Alzheimer's disease. J Am Med Dir Assoc 8, 21-30.

[24] Youn YC, Park KW, Han SH, Kim S (2011) Urine neural thread protein measurements in Alzheimer disease. $J$ Am Med Dir Assoc 12, 372-376.

[25] Albert MS, DeKosky ST, Dickson D, Dubois B, Feldman HH, Fox NC, Gamst A, Holtzman DM, Jagust WJ, Petersen RC, Snyder PJ, Carrillo MC, Thies B, Phelps CH (2011) The diagnosis of mild cognitive impairment due to Alzheimer's 
disease: Recommendations from the National Institute on Aging-Alzheimer's Association workgroups on diagnostic guidelines for Alzheimer's disease. Alzheimers Dement 7, 270-279.

[26] McKhann GM, Knopman DS, Chertkow H, Hyman BT, Jack CR Jr, Kawas CH, Klunk WE, Koroshetz WJ, Manly JJ, Mayeux R, Mohs RC, Morris JC, Rossor MN, Scheltens P, Carrillo MC, Thies B, Weintraub S, Phelps $\mathrm{CH}$ (2011) The diagnosis of dementia due to Alzheimer's disease: Recommendations from the National Institute on Aging-Alzheimer's Association workgroups on diagnostic guidelines for Alzheimer's disease. Alzheimers Dement 7, 263-269.

[27] Villemagne VL, Burnham S, Bourgeat P, Brown B, Ellis KA, Salvado O, Szoeke C, Macaulay SL, Martins R, Maruff P, Ames D, Rowe CC, Masters CL (2013) Amyloid beta deposition, neurodegeneration, and cognitive decline in sporadic
Alzheimer's disease: A prospective cohort study. Lancet Neurol 12, 357-367.

[28] Teipel S, Drzezga A, Grothe MJ, Barthel H, Chetelat G, Schuff N, Skudlarski P, Cavedo E, Frisoni GB, Hoffmann W, Thyrian JR, Fox C, Minoshima S, Sabri O, Fellgiebel A (2015) Multimodal imaging in Alzheimer's disease: Validity and usefulness for early detection. Lancet Neurol 14, 1037-1053.

[29] Tekin S, Mega MS, Masterman DM, Chow T, Garakian J, Vinters HV, Cummings JL (2001) Orbitofrontal and anterior cingulate cortex neurofibrillary tangle burden is associated with agitation in Alzheimer disease. Ann Neurol 49, 355361.

[30] de la Monte SM, Chen GJ, Rivera E, Wands JR (2003) Neuronal thread protein regulation and interaction with microtubule-associated proteins in SH-Sy5y neuronal cells. Cell Mol Life Sci 60, 2679-2691. 